\title{
A Novel Nine-Switch Inverter for Independent Control of Two Three-phase Loads
}

\author{
Tsutomu Kominami \\ Department of Electrical and Computer Engineering \\ Graduate School of Engineering \\ Yokohama National University \\ Email: kominami@fujilab.dnj.ynu.ac.jp
}

\author{
Yasutaka Fujimoto \\ Department of Electrical and Computer Engineering \\ Faculty of Engineering \\ Yokohama National University \\ Email: fujimoto@ynu.ac.jp
}

\begin{abstract}
Industrial applications require large numbers of motors. For example, motors are used to manipulate industrial robots, an electric vehicles with in-wheel motors and electric trains. Two methods exist for controlling PM motors providing an inverter to control each motor, and connecting the motors in parallel and driving them with a single inverter. The first method makes an experimental apparatus complex and expensive; the second does not allow independent control of each motor because of differences in rotor angle between the two motors. Thus, we propose a novel nine-switch inverter that can independently control two three-phase loads. This paper introduces the structure of the nine-switch inverter, which is made from nine switches. The validity of the proposed inverter is verified through simulations and experiments.
\end{abstract}

\section{INTRODUCTION}

The permanent magnet (PM) motor is finding widespread application because of its good efficiency. In particular, a variety of these motors are needed for factory applications, such as manipulating industrial robots. There are two methods of controlling dual PM motors: providing two separate inverters to drive each motor, and connecting the two motors in parallel and driving them with a single inverter. The first method increases the complexity and cost of the experimental apparatus. The second method cannot provide independent control of the two motors. Thus, we propose a novel nineswitch inverter that independently controls two three-phase loads. A similar concept called the five-leg inverter has been reported. This approach uses 10 switches, and its operational principle is verified through simulations[1].

This paper proposes a nine-switch inverter made from nine switches. This inverter eliminates three switching devices from the dual three-phase inverter[2][3][4][5]. The validity of the proposed inverter is verified through simulations and experiments.

\section{Structure of the Nine-Switch InVerter}

\section{A. Basic Concept}

Figure 1 shows the structure of the proposed nine-switch inverter, which consists of two three-phase inverters combined with three common switches (UM, VM, and WM). The upper portion in Fig. 1 is called Invl, and the lower part is called Inv2. Inv1 consists of switches $\mathrm{UH}, \mathrm{VH}, \mathrm{WH}, \mathrm{UM}, \mathrm{VM}$, and
WM, and Inv2 consists of the switches UM, VM, WM, UL, VL, and WL.

A pulse-width modulator (PWM) generates gate signals for Inv1 and Inv2, as shown in Fig. 2. This PWM modulator has a unique carrier waveform. From this waveform, a reference for Inv2 is lower than carrier2 if the PWM modulation for Inv1 is calculated. Moreover, the Invl reference exceeds carrierl if the PWM modulation for Inv2 is calculated. Therefore, switches UL, VL, and WL are in the ON state when InvI is driven (mode1) and switches $\mathrm{UH}, \mathrm{VH}$, and $\mathrm{WH}$ are in the ON state when Inv2 is driven (mode2). Figure 3 shows the state of each mode.

\section{B. Method of Realization}

The Carrierl and the carrier in Fig. 2 can be combined when generating the gate signals. Therefore, the PWM modulation of Invl is calculated at the upper part of a triangular wave, and the PWM modulation of Inv2 is calculated at its lower part, as shown in Fig. 4. Let a U-phase voltage reference for InvI be $V_{u 1}^{r e f}$, and a U-phase voltage reference for Inv2 be $V_{u 2}^{r e f}$. Assume that $V_{u 1}^{r e f}$ and $V_{u 2}^{r e f}$ are given by

$$
\begin{aligned}
V_{u 1}^{r e f} & =A_{1} \sin \left(2 \pi f_{1} t+\phi_{1}\right) \\
V_{u 2}^{r e f} & =A_{2} \sin \left(2 \pi f_{2} t+\phi_{2}\right)
\end{aligned}
$$

where $A_{1}, A_{2}$ are amplitudes, $f_{1}, f_{2}$ are frequencies, and $\phi_{1}$, $\phi_{2}$ are phases. A general modulation rate, $m$, is given by

$$
m=\frac{V^{r e f}}{E / 2}
$$




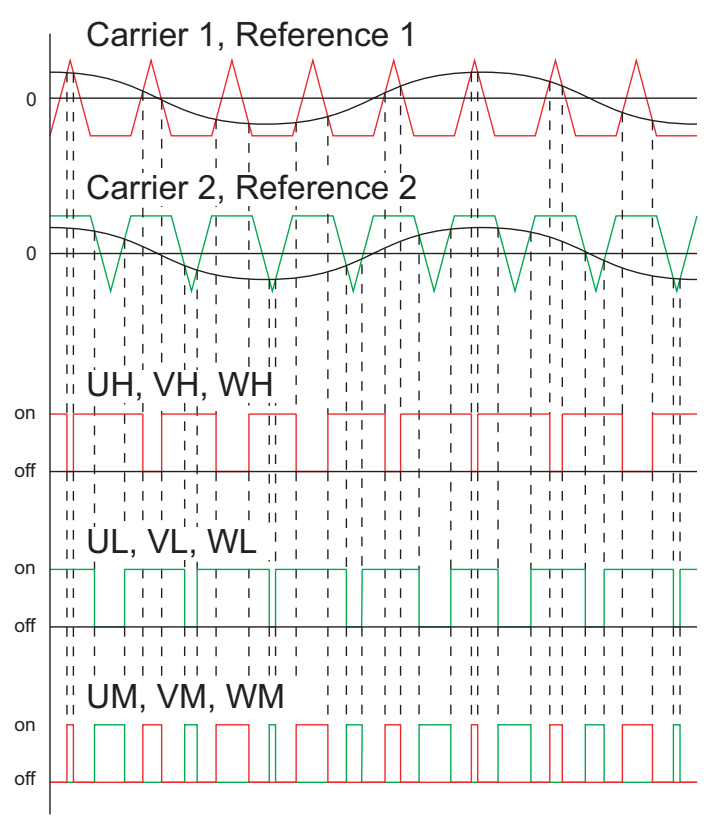

Fig. 2. Principle of operation.

where $E$ is a dc source voltage. An offset, $E / 4$, is added to the reference in (1) and an offset $-E / 4$ is added to the reference in (2) when calculating the proposed PWM modulation. Therefore,

$$
\begin{aligned}
& m_{1 u}=\frac{V_{u 1}^{r e f}+E / 4}{E / 2}=\frac{V_{u 1}^{r e f}}{E / 2}+\frac{1}{2} \\
& m_{2 u}=\frac{V_{u 2}^{r e f}-E / 4}{E / 2}=\frac{V_{u 2}^{r e f}}{E / 2}-\frac{1}{2} .
\end{aligned}
$$

From these transformations, the range of the references for Inv1 and Inv2 become $-E / 4 \leq V_{u 1}^{\text {ref }} \leq E / 4$ and $-E / 4 \leq$ $V_{u 2}^{r e f} \leq E / 4$, respectively. The gate signals for the switches $\mathrm{UH}, \mathrm{VH}$, and $\mathrm{WH}$ are positive logic values generated by the reference for InvI and the upper part of the carrier. The gate signals for switches UL, VL, and WL are negative logic values generated by the reference of Inv2 and the lower part of the carrier. The gate signals for the switches UM, VM, and WM are reversed values generated by the logical OR value of the gate signals for switches $\mathrm{UH}, \mathrm{VH}, \mathrm{WH}$ and $\mathrm{UL}, \mathrm{VL}, \mathrm{WL}$, as shown in Fig. 5.

\section{Improving Voltage Utilization}

The nine-switch inverter shares one dc voltage source between Inv1 and Inv2. Therefore, voltage utilization for InvI and $I n v 2$ is $50 \%$. However, this section proposes a method of improving voltage utilization. Each inverter's use of the voltage source changes with its reference value. Let the distribution rate of voltage utilization be $\alpha(0 \leq \alpha \leq 1)$. First, we derive an equation for a single phase. $\alpha$ is given by

$$
\alpha=\frac{A_{1}}{A_{1}+A_{2}} .
$$

An offset using this $\alpha$ is added to the variations of the reference for InvI and Inv2. The offsets are decided as modulation

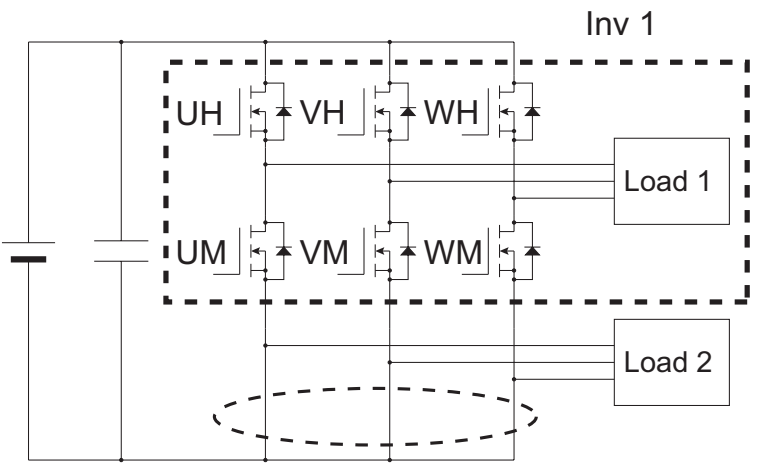

$\mathrm{UL}, \mathrm{VL}$, and $\mathrm{WL}$ are in $\mathrm{ON}$ state.

mode1

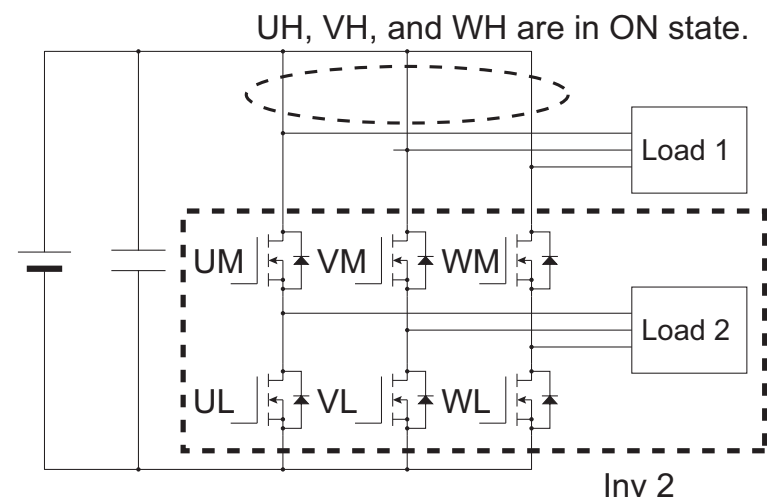

mode2

Fig. 3. Operation mode (mode1 and mode2).

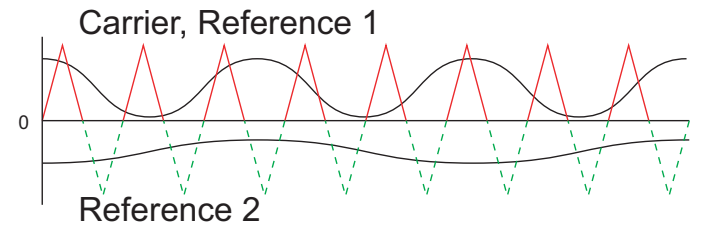

Fig. 4. PWM modulation of the novel inverter.

rates located on the center of the divided carrier. Thus, each offset is given by

$$
\begin{aligned}
& \text { offset }_{1}=1-\alpha \\
& \text { offset }_{2}=-\alpha .
\end{aligned}
$$

Therefore, the modulation rate of the U-phase is given by

$$
\begin{aligned}
& m_{1 u}=\frac{V_{u 1}^{r e f}}{E / 2}+1-\alpha \\
& m_{2 u}=\frac{V_{u 2}^{r e f}}{E / 2}-\alpha .
\end{aligned}
$$

Next, we derive an equation for three-phase operation. The maximal value of each absolute of the three-phase reference is represented by $r_{1}$ and $r_{2}$. Finally, the rate of apportionment 


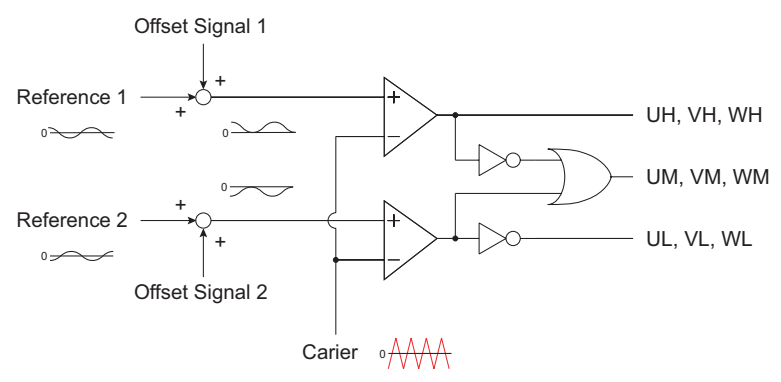

Fig. 5. Method of generation gate signals.

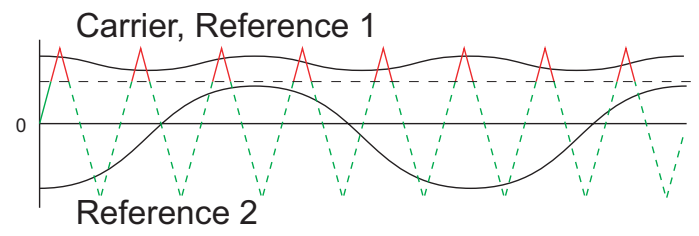

Fig. 6. PWM modulation with the distribution rate of voltage utilization.

$\alpha$ and the modulation rate are given by

$$
\begin{aligned}
& \alpha=\frac{\left|r_{1}\right|}{\left|r_{1}\right|+\left|r_{2}\right|} \\
& \mathbf{m}_{\mathbf{1}}=\frac{\mathbf{V}_{\mathbf{1}}^{\text {ref }}}{E / 2}+(1-\alpha) \mathbf{e} \\
& \mathbf{m}_{\mathbf{2}}=-\frac{\mathbf{V}_{\mathbf{2}}^{\text {ref }}}{E / 2}-\alpha \mathbf{e}
\end{aligned}
$$

where

$$
\begin{aligned}
\mathbf{V}_{\mathbf{i}}^{\text {ref }} & =\left[\begin{array}{lll}
V_{u i}^{r e f} & V_{v i}^{r e f} & V_{w i}^{r e f}
\end{array}\right]^{T} \\
\mathbf{m}_{\mathbf{i}} & =\left[\begin{array}{lll}
m_{u i} & m_{v i} & m_{w i}
\end{array}\right]^{T} \\
\mathbf{e} & =\left[\begin{array}{lll}
1 & 1 & 1
\end{array}\right]^{T} \\
i & =1,2 ;-1 \leq m_{i} \leq 1
\end{aligned}
$$

This method can improve voltage utilization of either Inv1 or Inv2. Figure 6 shows PWM modulation with the distribution rate of voltage utilization. Figure 7 shows a block diagram of the circuit that establishes the modulation rate.

\section{Simulation}

A simulation performed to verify the validity of the proposed inverter. In this simulation, we impose a different reference on each inverter. The reference for Invl is a threephase sine wave with an amplitude of 10 [V] and a frequency of $320[\mathrm{~Hz}]$, and the reference for Inv2 is a three-phase sine wave with an amplitude of 10 [V] and a frequency of 640 [Hz]. A three-phase LR load is connected to each inverter. Table I shows the simulation parameters. Figure 8 gives the U-phase current of Invl and Fig. 9 gives the U-phase current of Inv2. These results indicate that the nine-switch inverter can independently control amplitude and frequency for two loads.
TABLE I

PARAMETERS OF SIMULATIONS.

\begin{tabular}{ccc}
\hline DC source voltage & $E$ & $40[\mathrm{~V}]$ \\
Frequency of the carrier & $f$ & $10[\mathrm{kHz}]$ \\
Resistance & $R$ & $47[\Omega]$ \\
Inductance & $L$ & $15000[\mu \mathrm{H}]$ \\
\hline
\end{tabular}

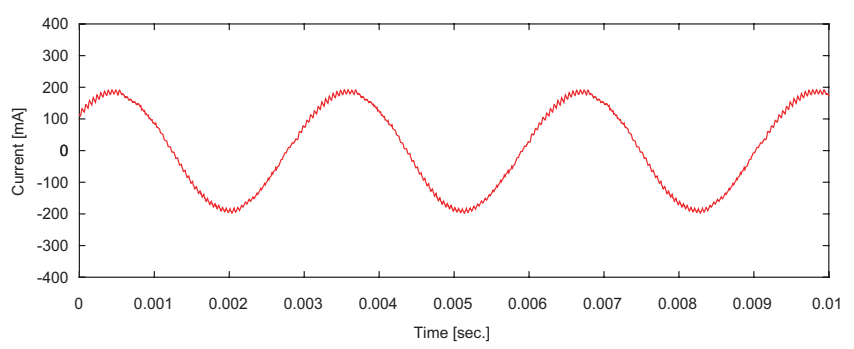

Fig. 8. Simulated current of Inv1 (U-phase). The reference for Inv1 is a three-phase sine wave with an amplitude of 10 [V] and a frequency of 320 [Hz].

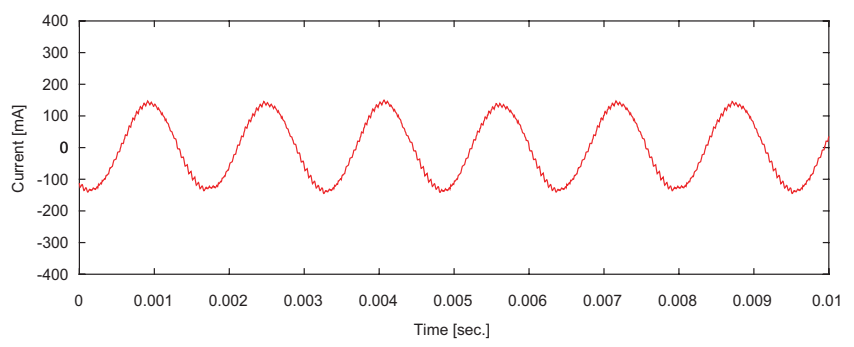

Fig. 9. Simulated current of Inv2 (U-phase). The reference for Inv2 is a three-phase sine wave with an amplitude of 10 [V] and a frequency of 640 $[\mathrm{Hz}]$.

\section{EXPERIMENT}

\section{A. Experiment Using LR Loads}

We conducted an experiment to verify the inverter's operation. The parameters of the experimental system are the same as for the simulation. The reference for Invl is a three-phase sine wave with an amplitude of 10 [V] and a frequency of $320[\mathrm{~Hz}]$; the reference for Inv2 is a three-phase sine wave with an amplitude of 10 [V] and a frequency of $640[\mathrm{~Hz}]$. Figure 10 shows the U-phase current for Inv1, and Fig. 11 shows the U-phase current for Inv2. These results demonstrate that the nine-switch inverter independently controls amplitude and frequency for two loads. However, the current waves have some ripples.

1) Independence of Inv1 and Inv2: To verify independent control, we conducted an experiment with different references for Inv1 and Inv2. In this experiment, the reference for Inv1 is a three-phase sine wave with an amplitude of 20 [V] and a frequency of $320[\mathrm{~Hz}]$, while the reference for Inv2 is zero volts. Figure 12 shows the U-phase current for Inv1, and Fig. 13 shows the U-phase current for Inv2. Figure 12 shows that Inv1 is driven correctly. However, as shown in Fig. 13, the current of Inv1 interferes with Inv2 by less than $1 \%$. In the future, we will examine the improvement of the ripple 


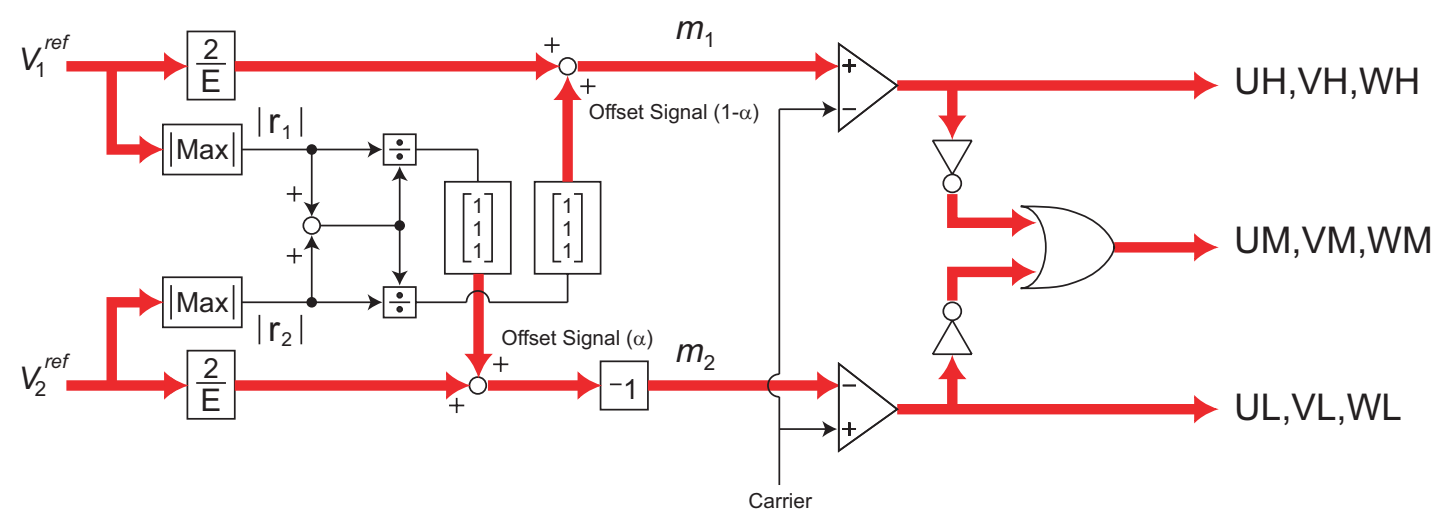

Fig. 7. Block diagram of establishment of modulation rate.

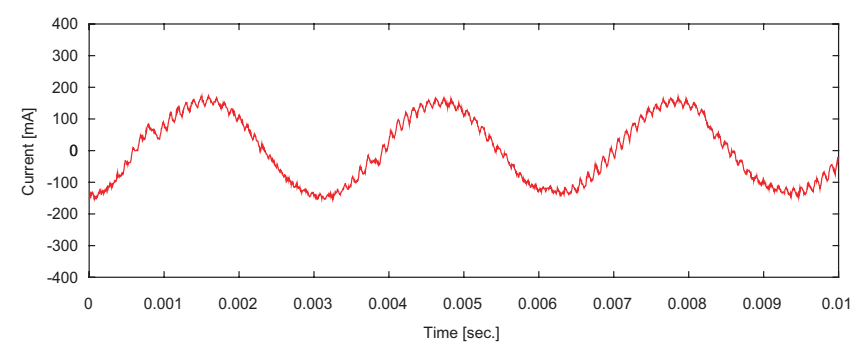

Fig. 10. Measured current of Inv1 (U-phase). The reference for Inv1 is a three-phase sine wave with an amplitude of $10[\mathrm{~V}]$ and a frequency of 320 $[\mathrm{Hz}]$.

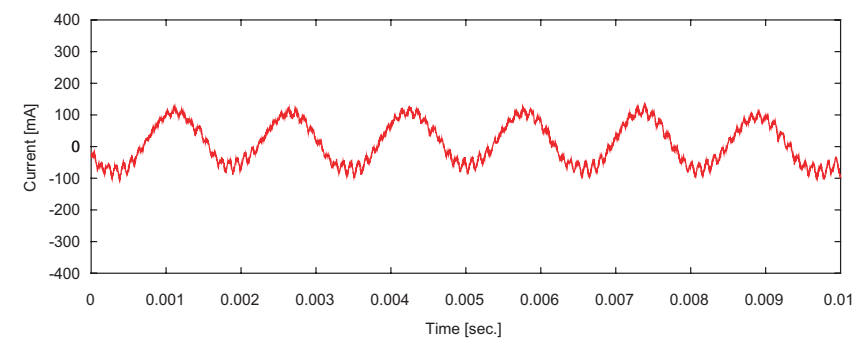

Fig. 11. Measured current of $\operatorname{Inv2}$ (U-phase). The reference for $\operatorname{Inv} 2$ is a three-phase sine wave with an amplitude of 10 [V] and a frequency of 640 $[\mathrm{Hz}]$.

amplitude and inverter interference.

\section{B. Experiment Using Permanent Magnet Synchronous Motor}

We conducted an experiment that connected two permanent magnet synchronous motors as loads. These motors had a rated output of 60 [W]. In this experiment, the Inv 1 reference is a three-phase sine wave with a frequency of $50[\mathrm{~Hz}]$ and the reference for Inv2 is a three-phase sine wave with a frequency of $30[\mathrm{~Hz}]$. These motors are driven with the frequencies of Inv1 and Inv2. Line voltages of the $\mathrm{U}-\mathrm{V}$ phase and $\mathrm{W}-\mathrm{V}$ phase for Inv1 are shown in Fig. 14 and those for Inv2 are shown in Fig. 15. These results confirm that the nine-switch inverter can independently control two motors.

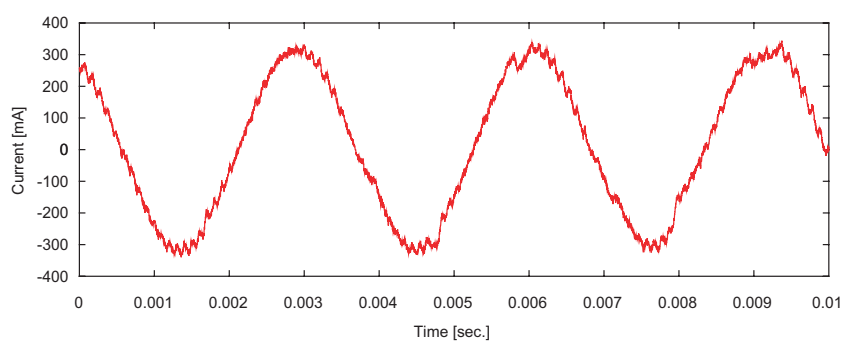

Fig. 12. Measured current of Inv1 (U-phase). The reference for Inv1 is a three-phase sine wave with an amplitude of $20[\mathrm{~V}]$ and a frequency of 320 $[\mathrm{Hz}]$.

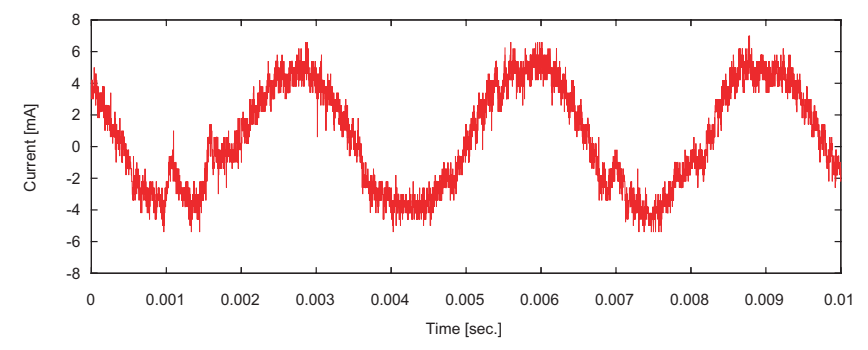

Fig. 13. Measured current of Inv2 (U-phase). The reference for Inv2 is zero volts.

\section{CONCLUSION}

This paper proposes a nine-switch inverter and a PWM method that can independently control two three-phase loads.

The simulations and the experiments were performed to verify the validity of the proposed inverter. The results confirmed that the nine-switch inverter can independently control amplitude and frequency for two three-phase loads, and parmanentmagnet synchronous motors; however, there is some ripple amplitude, and slight interference between Inv1 and Inv2. Work is needed to improve of the interference problem. 


\section{REFERENCES}

[1] Yusuke Nozawa, Motoki Hizume, Yuta Kimura, Kazuo Oka, and Kouki Matsuse, "Independent Position Control of Two Permanent Magnet Synchronous Motors with Five-Leg Inverter By the Expanded Two-Arm Modulation Method", IEEJ Industry Applications Society Conference, 2005, (in Japanese)

[2] Tsutomu Kominami and Yasutaka Fujimoto, "Magnetic Levitation Control and Spiral-Linear Transformation System for Spiral Motor", IEEE Int. Workshop on Advanced Motion Control, vol. 2, pp. 529-534, 2006

[3] Tsutomu Kominami and Yasutaka Fujimoto, "Proposal of a Nine-Switch Inverter That Can Independently Control Two PM Motors", IEEJ Industry Applications Society Conference, pp. 187-190, 2006, (in Japanese)

[4] Kazuo Oka and Kouki Matsuse, "A Nine-Switch Inverter for Driving Two AC Motors Independently", IEEJ Trans. on Electrical and Electronic Engineering, 2007

[5] Tsutomu Kominami and Yasutaka Fujimoto, "Development of a NineSwitch Inverter That Can Independently Control Two Loads", IEEJ Annual Meeting Record, pp. 133-134, 2007, (in Japanese)

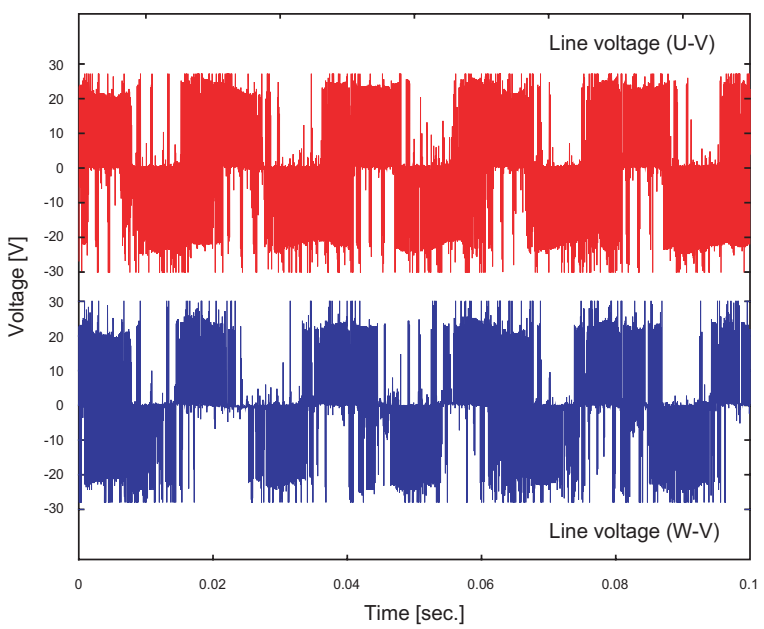

Fig. 14. Line voltage of U-V phase and $\mathrm{W}-\mathrm{V}$ phase; Inv1.

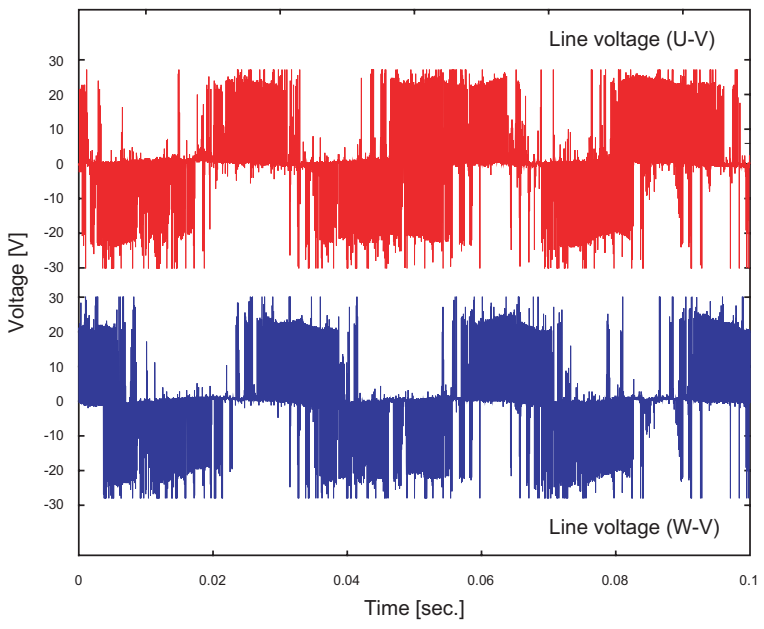

Fig. 15. Line voltage of U-V phase and W-V phase; Inv2. 